\title{
MiniVGGNet Kullanılarak Hiperspektral Görüntü Sınıflandırma
}

\section{Hyperspectral Image Classification Using MiniVGGNet}

\author{
Hüseyin FIRAT ${ }^{* 1}$ iD , Murat UÇAN ${ }^{1}$ (D) ${ }^{\text {Davut HANBAY2 }}{ }^{2}$ iD \\ 'Bilgisayar Teknolojileri Bölümü, Dicle Üniversitesi-Tenik Bilimler MYO, Diyarbakır, Türkiye \\ ${ }^{2}$ Bilgisayar Mühendisliği Bölümü, İnönü Üniversitesi, Malatya, Türkiye \\ (hossein.firat@gmail.com, murat.ucan@dicle.edu.tr, davut.hanbay@inonu.edu.tr)
}

Published: Oct.20, 2021

$\ddot{O}_{z}$ etçe - Hiperspektral görüntü sınıflandırma uzaktan algılanan görüntülerin analizinde yaygın olarak kullanılmaktadır. Son zamanlarda, derin öğrenme hiperspektral görüntü sınıflandırmasında en etkili yöntem olarak görülmektedir. Özellikle evrişimsel sinir ağları (ESA) bu alanda giderek daha fazla ilgi görmektedir. ESA, spektral, uzamsal veya spektral-uzamsal alanlardan hiperspektral görüntülerin daha soyut özelliklerini öğrenebilen otomatik yaklaşımlar sağlamaktadır. Bu çalı̧̧ma kapsamında, hiperspektral özellikler arasındaki ilişkilerden tam olarak yararlanmak ve sınıflandırma doğruluğunu arttırmak için 3B ESA tabanlı MiniVGGNet ağı önerilmektedir. 3B ESA ile spektral-uzamsal özellikler eş zamanlı olarak çıkarılmaktadır. MiniVGGNet ile de eğitilebilir parametre sayısı azaltılmakta ve eğitim süresi kısaltılmaktadır. Ayrıca, hiperspektral görüntülerin yüksek boyutluluğundan kaynaklanan hesaplama karmaşıklığını azaltmak için ön işleme yöntemi olarak temel bileşen analizi kullanılmaktadır. Önerilen yöntemin performansını test etmek için Indian Pines, Pavia Üniversitesi ve Salinas uzaktan algılama veri kümeleri üzerinde uygulamalar gerçekleştirilmiştir. Sonuçlar, farklı derin öğrenme tabanlı yöntemlerle karşılaştırılmıştır. Hiperspektral görüntü sınıflandırması için önerilen yöntem kullanılarak daha iyi sınıflandırma performansı elde edilmiştir.

Anahtar Kelimeler : Temel bileşen analizi, MiniVGGNet, Hiperspektral görüntü sinıflandırma, $3 B$ ESA.

Abstract - Hyperspectral image classification is widely used in the analysis of remote sensing images. Recently, deep learning has been seen as the most effective method for hyperspectral image classification. Especially, Convolutional neural networks (CNN) are getting more and more attention in this field. CNN provides automated approaches that can learn more abstract features of hyperspectral images from spectral, spatial or spectral-spatial fields. In this study, a 3D CNN based MiniVGGNet network is proposed to take full advantage of the relationships between hyperspectral features and to increase the classification accuracy. With 3D $\mathrm{CNN}$, spectral-spatial features are extracted simultaneously. With MiniVGGNet, the number of trainable parameters is reduced and the training time is shortened. In addition, principal component analysis (PCA) is used as a preprocessing method to reduce the computational complexity caused by the high dimensionality of hyperspectral images. In order to test the performance of the proposed method, applications were performed on remote sensing datasets of Indian Pines, University of Pavia and Salinas. The results were compared with different deep learning-based methods. Better classification performance is obtained by using the proposed method for hyperspectral image classification.

Keywords : Principal component analysis, MiniVGGNet, Hyperspectral image classification,3D CNN. 


\section{Giriş}

Hiperspektral sensörler, elektromanyetik spektrumun geniş bir bölümünde çok sayıda kanalda (bantlarda) spektral özellikleri ayırarak, Dünya yüzeyindeki nesnelerin ayrıntılı bilgilerini elde etmektedir. Bu zengin spektral bilgiler, yüzey malzemelerinin daha kapsamlı bir şekilde yorumlanması ve analizi için hiperspektral görüntülerin (HG) kullanılmasına izin vermektedir (Liu et al., 2020). Buna göre, hiperspektral uzaktan algılama, çevresel izleme, arazi yönetimi, tarım ve madencilik gibi çeşitli araştırma alanlarında yaygın olarak kullanılmaktadır (Jia et al., 2020; Firat and Hanbay, 2021). Bu uygulamalar arasında, görüntü sınıflandırma, $\mathrm{HG}^{\prime}$ deki her pikseli benzersiz bir anlamsal kategori veya sınıfa atamayı amaçlamaktadır. Bununla birlikte, HG'nin yüksek boyutluluğu, sınıflandırma için önemli bir soruna neden olmaktadır. HG sınıflandırma yöntemlerinin çoğu, yüksek boyutluluk için bir çözüm olarak boyut indirgeme yöntemlerini kullanmaktadır. HG sınıflandırmada boyut indirgeme yöntemleri temel olarak bant çıkarma ve bant seçimi olmak üzere ikiye ayrılmaktadır. Bant seçim yöntemi, HG'lerden en uygun bantları seçmekte ve fazla bantları ortadan kaldırmaktadır. Bant çıkarma, HG verilerini daha yüksek bir boyuttan daha düşük bir boyuta dönüştüren popüler boyut indirgeme yöntemidir. Çıkarılan bantların uzamsal boyutu, giriş HG'sinin uzamsal boyutuna tam olarak benzemektedir. Temel bileşen analizi (TBA), HG sınıflandırma için en yaygın olarak kullanılan bant çıkarma tekniğidir. TBA doğrusal bir tekniktir ve diğer tekniklere göre hesaplama açısından daha az karmaşıktır (Mohan and Meenakshi Sundaram, 2020).

Son yıllarda derin öğrenme tabanlı yöntemlerin gelişimi, HG sınıflandırması üzerinde büyük bir etki oluşturmaktadır. Gelişmiş özellik çıkarma yöntemleri gerektiren geleneksel yöntemlerle karşılaştırıldığında, derin öğrenme tabanlı yöntemler, özellikleri eğitim aşamasında otomatik olarak öğrenmekte ve sınıflandırma için kullanmaktadır. Mevcut derin öğrenme tabanlı yöntemler arasında tam bağlantılı ileri beslemeli sinir ağları (Chen et al., 2014), evrişimli sinir ağları (ESA) (Hanbay, 2020; Üzen et al., 2021) ve tekrarlayan sinir ağları (Mou et al., 2017) vb. bulunmaktadır. Bu ağlar arasında ESA, daha yüksek kalitede hiperspektral görüntü sınıflandırması için daha ayırt edici özellikler sağlayabilen güçlü bir özellik öğrenme özelliğine sahiptir. Bununla birlikte, ESA'lar hiperspektral görüntï sınıflandırması için en etkili yöntemlerden biri olduğu ve spektral tabanlı, uzamsal tabanlı ve spektral-uzamsal tabanlı dahil olmak üzere uygulamalarda farklı bakış açıları göstermektedirler (Paoletti et al., 2018). Spektral tabanlı yöntemler kavramsal olarak basit ve uygulaması kolaydır, çoğu tek boyutlu ESA (1B ESA) mimarilerini spektral bantlardan özellik çıkarmak için kullanmaktadır. Ancak, bu yöntemler spektral bağlamları göz ardı ederek yetersiz bir sınıflandırma performansı ile sonuçlanmaktadır (Li et al., 2019). Uzamsal tabanlı yaklaşımlar, iki boyutlu ESA (2B ESA) mimarilerini kullanarak uzamsal bilgileri çıkarmak için taramadaki belirli bir pikselin komşu piksellerini dikkate almaktadır. Uzamsal bağlamlara daha fazla odaklandıklarından, bu yöntemlerin çoğu, ilk önce görüntü boyutunu azaltmak için TBA ve oto-kodlayıcı gibi ön işleme yöntemlerini kullanmaktadır. Uzamsal özellikler ve spektral özellikler ayrı ayrı çıkarıldıklarından, sınıflandırma için önemli olabilecek ortak uzamsal spektral korelasyon bilgilerinden tam olarak yararlanamayabilirler. Bu işlem spektrumdaki bazı bilgileri kaybedecek ve bu yöntemlerin farklı nesneleri ayırt etme kapasitesini azaltacaktır (Huang and Chen, 2020). Uzamsal-spektral yaklaşımlar, üç boyutlu ESA (3B ESA) mimarilerini kullanarak, uzamsal ve spektral bilgileri birleştirmektedir. 3B ESA yöntemi, ağ modelinin girdisi olarak komşuluk bloğunu seçmektedir. Aynı zamanda daha iyi sınıflandırma doğruluğu elde etmek için eşzamanlı olarak orijinal hiperspektral görüntülerden spektral ve uzamsal özellikleri çıkarmaktadır. Hiperspektral küplerin hem uzamsal hem de spektral alanlarındaki özellikleri ögrenebildikleri için, hiperspektral görüntü sınıflandırma kalitesini iyileştirmek için bu yöntemler giderek daha fazla ilgi görmektedir (Chen, Y.; Jiang, H.; Li, C.; Jia, X.; Ghamisi, 2016). Bu çalışma kapsamında kolayca eğitilebilen 3B ESA tabanlı MiniVGGNet adlı daha küçük bir VGGNet mimarisi önerilmektedir. Bu çalışmanın ana katkıları şu şekildedir:

1. Önerilen 3B ESA tabanlı MiniVGGNet, yüksek sınıflandırma doğruluğu sağlarken, eğitilebilir parametre sayısını ve eğitim-test sürelerini düşürmektedir.

2. 3B ESA ile spektral ve uzamsal özellikler eşzamanlı olarak çıkarıldığından, 3 boyutlu hiperspektral görüntülerin yapısal özelliklerinden tam olarak yararlanılmaktadır.

3. HG'lerinin yüksek boyutluluğundan/spektral bant fazlalı̆̆ından oluşan hesaplama karmaşıklığını azaltmak için boyut indirgeme yöntemi olarak TBA kullanılmaktadır. 
$\mathrm{Bu}$ çalışmanın geri kalanının düzeni şu şekildedir. Bölüm 2'de önerilen yöntem, Bölüm 3'te uygulamalarda kullanılan verisetleri ve elde edilen uygulama sonuçları yer almaktadır. Bölüm 4'te ise makalenin genel bir özeti verilmektedir.

\section{2. Önerilen Yöntem}

Önerilen Yöntem Şekil 1'de gösterildiği gibi üç bölümden oluşmaktadır. (1) Boyut indirgeme, (2) Örnek oluşturma ve (3) Özellik çıkarma ve sınıflandırma. İlk olarak, Hiperspektral görüntï $X, M \times$ $N \times D$ boyutunda 3 boyutlu bir küp olarak belirtilmiştir. Burada $M$ ve $N$, görüntünün uzamsal genişliği ve yüksekliğidir. $D$ ise, spektral bantların sayısını belirtmektedir. Yüzlerce spektral banttan oluşan HG'nin analizini kolaylaştırmak ve hesaplama karmaşıklığını azaltmak için spektral bant fazlalığını gidermek gerekmektedir. Bu doğrultuda, ilk önce orijinal HG verilerine spektral bantlar boyunca geleneksel TBA uygulanmaktadır. TBA, aynı uzamsal boyutları (yani, genişlik M ve yükseklik N) korurken spektral bant sayısını D'den B'ye düşürür. Herhangi bir nesneyi tanımak için çok önemli olan uzamsal bilgiyi koruyacak şekilde yalnızca spektral bantları azaltılmış oldu. Böylelikle, istenilen bant sayısına indirgenmiş oldu. İkinci olarak, görüntü sınıflandırma tekniklerini kullanmak için, hiperspektral veri küpü, merkezi piksele dayalı gerçek etiketlerin oluşturulduğu küçük üst üste binen 3B uzamsal parçalara bölünmüştür. Uzamsal konumda $(\mathrm{a}, \mathrm{b})$ merkezlenmiş ve $S x S$ penceresini veya uzamsal boyutu ve tüm $B$ spektral bantlarını kapsayan hiperspektral veri küpünden 3B komşu parçaları $S X S X B$ oluşturuldu. Hiperspektral veri küpünden üretilen toplam 3B parça sayısı $(n),(M-S+$ 1) $x(N-S+1)$ ile bulunur. Son olarak, oluşturulan $3 \mathrm{~B}$ hiperspektral veri küpü, özellik çıkarma ve sınıflandırma için 3B ESA tabanlı MiniVGGNet ağının girişine verilmektedir. 3B ESA, 2B ESA'da 2B evrişim yerine $3 \mathrm{~B}$ evrişim gerçekleştiren $2 \mathrm{~B}$ ESA modellerinin değiştirilmiş şeklidir. Evrişim işlemleri, 3B verilere uygulandığında hem uzamsal hem de spektral boyutlardan özelliklerin yakalanması istenilmektedir. Bu amaçla, 3B giriş verilerinden ortak uzamsal spektral özellikleri hesaplamak için 3B evrişim işlemlerinin 3B özellik küplerine uygulandığı 3B ESA kullanılmaktadır. 3B evrişim, 3B spektral görüntülerden uzamsal-spektral özelliklerin çıkarılmasına yardımcı olmaktadır. MiniVGGNet ağı ise, 6 evrişim, 3 havuzlama, düzleştirme, tam bağlantılı ve softmax katmanlarından oluşmaktadır. Tüm evrişim katmanlarında $3 \times 3 \times 3$ çekirdek boyutu kullanılmaktadır. Evrişim katmanlarında kullanılan filtreler ise, 1 ve 2 . Evrişim katmanlarında 32,3 ve 4. Evrişim katmanlarında 64, 5 ve 6 . katmanlarda ise 128 olarak alınmıştır. Ağ yapısında kullanılan tüm havuzlama katmanları maksimum havuzlamadır ve $2 \times 2 \times 2$ çekirdek boyutundadır. Her evrişim katmanından sonra bacth normalization (BN) ve RELU aktivasyon fonksiyonu kullanılmaktadır. A ğı daha düzenli hale getirmek ve öğrenme sürecinin aşırı uyum göstermesini önlemek için BN kullanılmıştır. Özellikle gradyan azalması ile eğitim süresi açısından RELU'lar diğer aktivasyon fonksiyonlarından daha hızlı olma eğilimindedir. Bundan dolayı, çalışma kapsamında aktivasyon fonksiyonu olarak RELU kullanılmıştır. Evrişim ve maksimum havuzlama katmanlarından sonra çıkarılan özellikler düzleştirilir ve sınıflandırma için tam bağlı katmanlara girdi olarak verilir. Bu yöntemde, 1024 nöron bulunan tam bağlantılı katman kullanılmaktadır. Aşırı öğrenmeyi önlemek için, tamamen bağlı katmandan sonra \%0.5 dropout oranına sahip dropout katmanı uygulanmaktadır. Tam bağlantılı katmanının (FC) çıktısı, gerekli sınıflandırma sonucunu oluşturmak için basit softmax sınıflandırıcıya verilmektedir. Önerilen yöntem ile ilgili daha fazla ayrıntı Tablo 1'de gösterilmektedir. Indian pines veriseti için önerilen yöntemdeki toplam eğitilebilir ağırlık parametresi sayısı 1.925.296'dır.

\section{Verisetleri ve Uygulama Sonuçları}

\subsection{Verisetleri:}

Önerilen yöntemin etkinliğini göstermek için Indian Pines (IP), Salinas (SA) ve Pavia Üniversitesi (PU) veri setlerini içeren üç gerçek hiperspektral veri kümesi kullanılmıştır. Indian pines, Pavia üniversitesi ve Salinas verisetleri http://www.ehu.eus/ccwintco/index.php/Hyperspectral_Remote_Sensing_Scenes websitesinden alınmaktadır. IP veri kümesinde 145x145 uzamsal boyutunda 400 ile 2500 nanometre (nm) dalga boyu aralığında toplam 224 spektral bant içeren görüntüler bulunmaktadır. Bunların arasından su emme bölgesini kapsayan 24 spektral bant atılmıştır. Toplam 200 bant sınıflandırma amaçlı kullanılacaktır. IP veri seti 16 tür arazi örtüsü sınıfı ve 10249 örnek içermektedir. PU veriseti, 430 ile $860 \mathrm{~nm}$ dalga boyu 
aralığında 103 spektral bant ile 610x340 uzamsal boyut piksellerinde oluşmaktadır. PU veriseti 9 sinıfa ayrilmakta ve 42776 örnek içermektedir. SA verisetinde, 512x217 uzamsal boyut ve 360 ile $2500 \mathrm{~nm}$ dalga boyu aralığında 224 spektral bant içeren görüntüler yer almaktadır. Su emici 20 spektral bant atıldıktan sonra toplam 204 bant sınıflandırma amaçlı kullanılacaktır. Bu veri kümesinde toplam 16 sınıf ve 54129 örnek bulunmaktadır. Verisetleri ile ilgili detaylı bilgi Tablo 2'de gösterilmektedir.

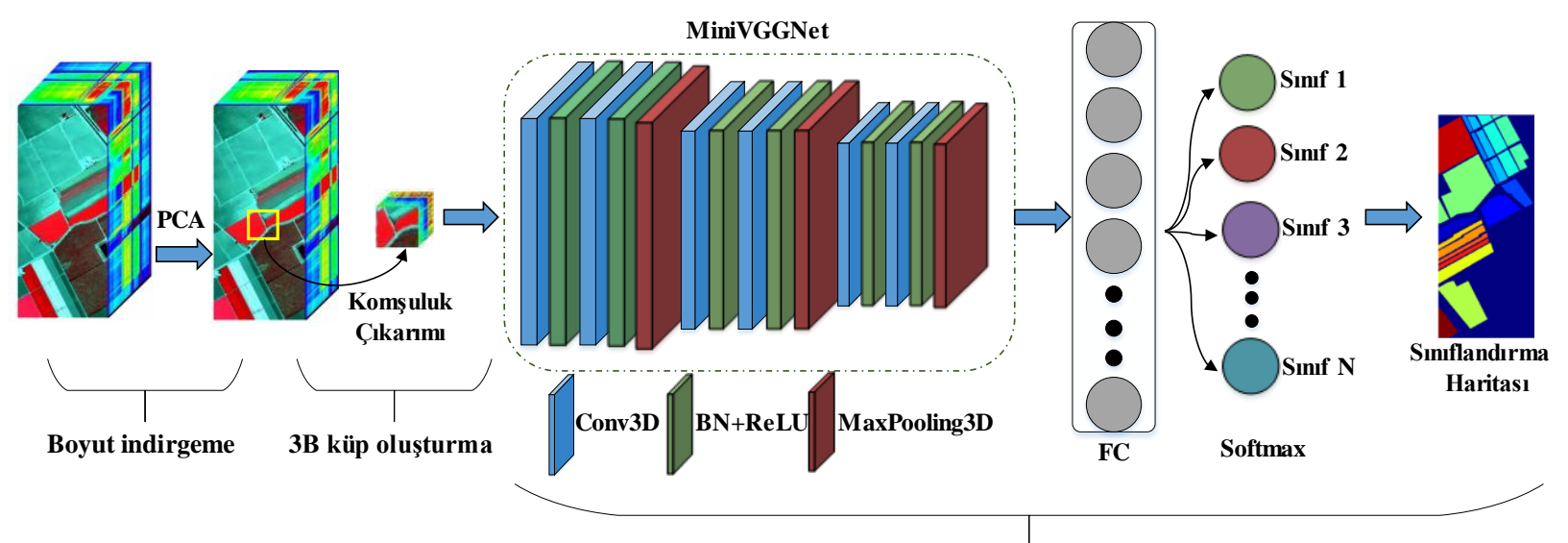

Özellik çıkarma ve sınıflandırma için 3B ESA tabanı MiniVGGNet

Şekil 1. Önerilen Yöntem

Tablo 1. Indian pines veriseti için önerilen yöntemin özeti

\begin{tabular}{ccc}
\hline Katman & Çıkıs Boyutu & Parametre Sayısı \\
\hline input_1 (Giriş katmanı) & $(15,15,15,1)$ & 0 \\
conv3d_1(Conv3d) & $(15,15,15,32)$ & 896 \\
BN_1 & $(15,15,15,32)$ & 128 \\
conv3d_2(Conv3d) & $(15,15,15,32)$ & 27680 \\
BN_2 & $(15,15,15,32)$ & 128 \\
Max_pooling3d_1 & $(8,8,8,32)$ & 0 \\
conv3d_3 (Conv3d) & $(8,8,8,64)$ & 55360 \\
BN_3 & $(8,8,8,64)$ & 256 \\
conv3d_4 (Conv3d) & $(8,8,8,64)$ & 110656 \\
BN_4 & $(8,8,8,64)$ & 256 \\
Max_pooling3d_2 & $(4,4,4,64)$ & 0 \\
conv3d_5(Conv3d) & $(4,4,4,128)$ & 221312 \\
BN_5 & $(4,4,4,128)$ & 512 \\
conv3d_6(Conv3d) & $(4,4,4,128)$ & 442496 \\
BN_6 & $(4,4,4,128)$ & 512 \\
Max_pooling3d_3 & $(2,2,2,128)$ & 0 \\
Flatten_1 & 1024 & 0 \\
Dense_1 & 1024 & 1049600 \\
Dropout_1 & 1024 & 0 \\
Dense_2 & 16 & 16400 \\
\hline
\end{tabular}

Toplam parametre sayısı: 1.926 .192

Ĕgitilebilir parametre sayısı: 1.925 .296 
Tablo 2. Kullanılan verisetleri ile ilgili bilgiler

\begin{tabular}{|c|c|c|c|c|c|c|}
\hline \multirow[b]{2}{*}{ No } & \multicolumn{2}{|c|}{ Indian Pines (IP) } & \multicolumn{2}{|l|}{ Salinas (SA) } & \multicolumn{2}{|c|}{ Pavia University (PU) } \\
\hline & Classes & Samples & Classes & Samples & Classes & Samples \\
\hline 1 & Alfalfa & 46 & Brocoli_green_weeds_1 & 2009 & Asphalt & 6631 \\
\hline 2 & Corn-notill & 1428 & Brocoli_green_weeds_2 & 3726 & Meadows & 18,649 \\
\hline 3 & Corn-mintill & 830 & Fallow & 1976 & Gravel & 2099 \\
\hline 4 & Corn & 237 & Fallow_rough_plow & 1394 & Trees & 3064 \\
\hline 5 & Grass-pasture & 483 & Fallow_smooth & 2678 & $\begin{array}{l}\text { Painted metal } \\
\text { sheets }\end{array}$ & 1345 \\
\hline 6 & Grass-trees & 730 & Stubble & 3959 & Bare soil & 5029 \\
\hline 7 & $\begin{array}{l}\text { Grass-pasture- } \\
\text { mowed }\end{array}$ & 28 & Celery & 3579 & Bitumen & 1330 \\
\hline 8 & Hay-windrowed & 478 & Grapes_untrained & 11,271 & $\begin{array}{l}\text { Self-blocking } \\
\text { Bricks }\end{array}$ & 3682 \\
\hline 9 & Oats & 20 & Soil_vinyard_develop & 6203 & Shadows & 947 \\
\hline 10 & Soybean-notill & 972 & Corn_senesced_green_weeds & 3278 & & \\
\hline 11 & Soybean-mintill & 2455 & Lettuce_romaine_t $4 \mathrm{wk}$ & 1068 & & \\
\hline 12 & Soybean-clean & 593 & Lettuce_romaine_5wk & 1927 & & \\
\hline 13 & Wheat & 205 & Lettuce_romaine_6wk & 916 & & \\
\hline 14 & Woods & 1265 & Lettuce_romaine_7wk & 1070 & & \\
\hline 15 & $\begin{array}{l}\text { Buildings-grass- } \\
\text { trees-drives }\end{array}$ & 386 & Vinyard_untrained & 7268 & & \\
\hline \multirow[t]{2}{*}{16} & Stone-steel-towers & 93 & Vinyard_vertical_trellis & 1807 & & \\
\hline & Total Number & 10249 & & 54129 & & 42776 \\
\hline
\end{tabular}

\subsection{Uygulama Sonuçları:}

Tüm uygulamalar çevrim içi bir platform olan Google Colab'da gerçekleştirilmektedir. Colab, iyi bir internet hızı gerektirmektedir. Donanım hızlandırıcı olarak grafik işlem birimi (GPU) ve Tensör işlem birimi (TPU)'yu sunmaktadır. Uygulamalarımızdaki kodlar Tensör İşlem Birimi (TPU), 35 GB Rasgele Erişim Belleği (RAM) ve veri hesaplaması için 107,77 GB depolama alanı içeren python 3 masaüstü bilgisayarda çalıştırılmıştır. Tüm uygulamalarda, eğitim-test oranı \%70-30 olarak alınmıştır. Optimizasyon için 1e-06 bozunma işlevine sahip Adam optimizer ve 0.001 öğrenme oranına sahip kategorik çaprazentropi kullanılmıştır. Eğitim işlemi, 256 batch size ve 100 epochs boyunca tekrar etmektedir. Softmax'ın kullanıldığı son katman hariç tüm katmanlar için bir aktivasyon işlevi olarak RELU kullanılmaktadır. Tüm verisetlerinde, evrişim için 15x15 komşuluk çıkarımı kullanılmaktadır. Bununla birlikte, TBA tekniğinden sonra çıkarılan bantların sayısı her iki veriseti için 15 temel bileşen kullanmaktadır. Uygulamalarda karşılaştııılan yöntemlerin sınıflandırma doğruluklarını değerlendirmek için genel doğruluk (GD), ortalama doğruluk (OD) ve kappa istatistiği (K) kullanılmıştır. GD, test verilerindeki doğru sınıflandırılmış örnekler ile toplam test örneği sayısı arasındaki oranla hesaplanmıştır. OD her kategorinin doğruluğunun ortalama değeridir. K, temel gerçek (ground truth) haritası ile sınıflandırma haritası arasında güçlü bir anlaşma ile ilgili karşılıklı bilgi sağlayan bir istatistiksel ölçüm metriğidir. Önerilen yöntem (ÖY) literatürden, 2B ESA (Makantasis et al., 2015), 3B ESA (Ben Hamida et al., 2018), M3B-ESA (3 boyutlu derin evrişimsel sinir ağı) (Mingyi He, Bo Li, 2017), SSRN (spektral-uzamsal artık ağ) (Zhong et al., 2018) ve HybridSN (Hybrid Spektral Network) (Roy et al., 2019) gibi derin öğrenmeye dayalı HG sınıflandırma yöntemleri ile karşılaştırılmıştır. Karşılaştırma sonuçlarının sınıflandırma doğrulukları IP veriseti için Tablo 3'te, PU veriseti için Tablo 4'te ve SA veriseti için Tablo 5'te gösterilmektedir. 
Tablo 3. IP veriseti için sınıflandırma sonuçları(\%)

\begin{tabular}{ccccccc}
\hline Sinıf & 2B-ESA & 3B-ESA & M3B-ESA & SSRN & $\begin{array}{c}\text { Hybrid } \\
\text { SN }\end{array}$ & ÖY \\
\hline $\mathbf{1}$ & 75,00 & 79,23 & 97,03 & 97,82 & 99,38 & $\mathbf{1 0 0}$ \\
$\mathbf{2}$ & 81,40 & 88,60 & 97,90 & 99,17 & 99,58 & $\mathbf{1 0 0}$ \\
$\mathbf{3}$ & 87,60 & 85,81 & 92,41 & 99,53 & 99,66 & $\mathbf{1 0 0}$ \\
$\mathbf{4}$ & 62,04 & 90,53 & 93,25 & 97,79 & 99,88 & $\mathbf{1 0 0}$ \\
$\mathbf{5}$ & 92,30 & 96,11 & 95,00 & 99,24 & 99,53 & $\mathbf{1 0 0}$ \\
$\mathbf{6}$ & 99,21 & 98,43 & 99,74 & 99,51 & $\mathbf{9 9 , 9 6}$ & 99,54 \\
$\mathbf{7}$ & 75,00 & 92,36 & 100 & 98,70 & 99,00 & $\mathbf{1 0 0}$ \\
$\mathbf{8}$ & 100 & 98,51 & 99,99 & 99,85 & 100 & $\mathbf{1 0 0}$ \\
$\mathbf{9}$ & 64,28 & 88,90 & 96,61 & 98,50 & 100 & $\mathbf{1 0 0}$ \\
$\mathbf{1 0}$ & 82,79 & 87,72 & 96,32 & 98,74 & 99,56 & $\mathbf{9 9 , 6 5}$ \\
$\mathbf{1 1}$ & 91,27 & 91,42 & 97,13 & 99,30 & $\mathbf{9 9 , 8 4}$ & 99,60 \\
$\mathbf{1 2}$ & 82,89 & 90,04 & 97,16 & 98,43 & 99,52 & $\mathbf{1 0 0}$ \\
$\mathbf{1 3}$ & 99,30 & 99,00 & 99,60 & 100 & 99,86 & $\mathbf{1 0 0}$ \\
$\mathbf{1 4}$ & 98,87 & 97,95 & 98,42 & 99,31 & 100 & $\mathbf{1 0 0}$ \\
$\mathbf{1 5}$ & 86,29 & 82,57 & 83,31 & 99,20 & 99,85 & $\mathbf{1 0 0}$ \\
$\mathbf{1 6}$ & $\mathbf{1 0 0}$ & 98,51 & $\mathbf{1 0 0}$ & 97,82 & 98,46 & $\mathbf{1 0 0}$ \\
\hline GD & $89,48 \pm 0,2$ & $91,10 \pm 0,4$ & $95,32 \pm 0,1$ & $99,19 \pm 0,3$ & $99,75 \pm 0,1$ & $\mathbf{9 9 , 8 3} \pm \mathbf{0 0 , 0}$ \\
OD & $86,14 \pm 0,8$ & $91,58 \pm 0,2$ & $96,41 \pm 0,7$ & $98,93 \pm 0,6$ & $99,63 \pm 0,2$ & $\mathbf{9 9 , 9 2} \pm \mathbf{0 , 0}$ \\
K & $87,96 \pm 0,5$ & $89,98 \pm 0,5$ & $94,70 \pm 0,2$ & $99,07 \pm 0,3$ & $99,71 \pm 0,1$ & $\mathbf{9 9 , 8 1} \pm \mathbf{0 , 0}$ \\
\hline
\end{tabular}

Tablo 4. PU veriseti için sınıflandırma sonuçları(\%)

\begin{tabular}{ccccccc}
\hline Sinıf & 2B-ESA & 3B-ESA & $\begin{array}{c}\text { M3B- } \\
\text { ESA }\end{array}$ & SSRN & HybridSN & ÖY \\
\hline $\mathbf{1}$ & 98,51 & 98,40 & 98,31 & 100 & 100 & $\mathbf{1 0 0}$ \\
$\mathbf{2}$ & 99,54 & 96,91 & 96,10 & 99,87 & 100 & $\mathbf{1 0 0}$ \\
$\mathbf{3}$ & 84,62 & 97,05 & 96,34 & 100 & 100 & $\mathbf{1 0 0}$ \\
$\mathbf{4}$ & 98,04 & 98,84 & 98,82 & 100 & 99,84 & $\mathbf{1 0 0}$ \\
$\mathbf{5}$ & 100 & 100 & 99,97 & 100 & 100 & $\mathbf{1 0 0}$ \\
$\mathbf{6}$ & 97,10 & 99,32 & 99,83 & 100 & 100 & $\mathbf{1 0 0}$ \\
$\mathbf{7}$ & 95,05 & 98,92 & 99,66 & 100 & 100 & $\mathbf{1 0 0}$ \\
$\mathbf{8}$ & 96,39 & 98,33 & 99,23 & 99,34 & 99,98 & $\mathbf{1 0 0}$ \\
$\mathbf{9}$ & 99,69 & 99,90 & 99,92 & 100 & 99,90 & $\mathbf{1 0 0}$ \\
\hline GD & $97,86 \pm 0,2$ & $96,53 \pm 0,1$ & $95,76 \pm 0,2$ & $99,90 \pm 0,0$ & $99,98 \pm 0,0$ & $\mathbf{1 0 0} \pm \mathbf{0 , 0}$ \\
OD & $96,55 \pm 0,0$ & $97,57 \pm 1,3$ & $95,08 \pm 1,2$ & $99,91 \pm 0,0$ & $99,97 \pm 0,0$ & $\mathbf{1 0 0} \pm \mathbf{0 , 0}$ \\
$\mathbf{K}$ & $97,16 \pm 0,5$ & $95,51 \pm 0,2$ & $94,50 \pm 0,2$ & $99,87 \pm 0,0$ & $99,98 \pm 0,0$ & $\mathbf{1 0 0} \pm \mathbf{0 , 0}$ \\
\hline
\end{tabular}


Tablo 5. SA veriseti için sınıflandırma sonuçları(\%)

\begin{tabular}{ccccccc}
\hline SinIf & 2B-ESA & 3B-ESA & M3B-ESA & SSRN & HybridSN & ÖY \\
\hline $\mathbf{1}$ & 100 & 98,41 & 97,50 & 100 & 100 & $\mathbf{1 0 0}$ \\
$\mathbf{2}$ & 99,96 & 100 & 100 & 100 & 100 & $\mathbf{1 0 0}$ \\
$\mathbf{3}$ & 99,63 & 99,23 & 99,43 & 100 & 100 & $\mathbf{1 0 0}$ \\
$\mathbf{4}$ & 99,28 & 99,90 & 99,51 & 99,89 & 100 & $\mathbf{1 0 0}$ \\
$\mathbf{5}$ & 99,20 & 99,43 & 99,72 & 100 & 100 & $\mathbf{1 0 0}$ \\
$\mathbf{6}$ & 100 & 99,55 & 99,23 & 100 & 100 & $\mathbf{1 0 0}$ \\
$\mathbf{7}$ & 100 & 99,72 & 99,45 & 100 & 100 & $\mathbf{1 0 0}$ \\
$\mathbf{8}$ & 93,62 & 89,75 & 92,63 & 100 & 100 & $\mathbf{1 0 0}$ \\
$\mathbf{9}$ & 100 & 99,81 & 99,70 & 100 & 100 & $\mathbf{1 0 0}$ \\
$\mathbf{1 0}$ & 98,82 & 98,36 & 97,31 & 99,91 & 100 & $\mathbf{1 0 0}$ \\
$\mathbf{1 1}$ & 99,73 & 98,12 & 98,05 & 100 & 100 & $\mathbf{1 0 0}$ \\
$\mathbf{1 2}$ & 100 & 98,96 & 98,50 & 100 & 100 & $\mathbf{1 0 0}$ \\
$\mathbf{1 3}$ & 100 & 98,93 & 98,70 & 100 & 100 & $\mathbf{1 0 0}$ \\
$\mathbf{1 4}$ & 99,86 & 98,60 & 98,42 & 100 & 100 & $\mathbf{1 0 0}$ \\
$\mathbf{1 5}$ & 91,52 & 79,31 & 87,18 & 99,96 & 100 & $\mathbf{1 0 0}$ \\
$\mathbf{1 6}$ & 99,92 & 94,51 & 91,11 & 100 & 100 & $\mathbf{1 0 0}$ \\
\hline GD & $97,38 \pm 0,0$ & $93,96 \pm 0,2$ & $94,79 \pm 0,3$ & $99,98 \pm 0,1$ & $100 \pm 0,0$ & $\mathbf{1 0 0} \pm \mathbf{0 , 0}$ \\
$\mathbf{O D}$ & $98,84 \pm 0,1$ & $97,01 \pm 0,6$ & $96,25 \pm 0,6$ & $99,97 \pm 0,0$ & $100 \pm 0,0$ & $\mathbf{1 0 0} \pm \mathbf{0 0 0}$ \\
$\mathbf{K}$ & $97,08 \pm 0,1$ & $93,32 \pm 0,5$ & $94,20 \pm 0,2$ & $99,97 \pm 0,1$ & $100 \pm 0,0$ & $\mathbf{1 0 0} \pm \mathbf{0 , 0}$ \\
\hline
\end{tabular}

Tablo 3, 4 ve 5, sırasıyla IP, PU ve SA verisetinden elde edilen çeşitli yöntemlerin sınıf bazlı ve GD, $\mathrm{OD}, \mathrm{K}$ olmak üzere üç değerlendirme metriğine göre sınıflandırma sonuçlarını vermektedir. Tablo 3'de 6 ve 11 nolu sınıflar haricinde diğer tüm sınıflarda önerilen yöntem ile en iyi sınıflandırma doğruluğu elde edilmiştir. Tablo 4 ve Tablo 5'te ise tüm sınıflarda \%100 sinıflandırma doğruluğu elde edilmiştir. GD, OD ve K değerlendirme metrikleri göz önüne alındığında önerilen yöntem her üç verisetinde de en iyi sınıflandırma performansına sahip olduğu Tablo 3,4 ve 5 'te görülmektedir. Önerilen yönteme en yakın sonuçlar her üç verisetinde de HybridSN yönteminde elde edilmiştir. Ayrıca PU ve SA verisetlerinde SSRN yöntemi de önerilen yönteme oldukça yakın sınıflandırma sonucu verdiği görülmektedir. Her üç verisetinin eğitim ve test süreleri Tablo 6'da gösterilmektedir. Eğitim süresi dakika (dk) ve test süresi saniye (sn) olarak ölçülmektedir. Önerilen yöntemin spektral-uzamsal tabanlı olan 3B ESA, SSRN ve HybridSN yöntemlerinden daha kısa sürede daha iyi sınıflandırma performansı elde ettiği görülmektedir.

Tablo 6. IP, PU ve SA veriseti için eğitim (dk) ve test (sn) süreleri

\begin{tabular}{ccccccc}
\hline \multirow{2}{*}{ Methods } & \multicolumn{2}{c}{ IP } & \multicolumn{2}{c}{ PU } & \multicolumn{2}{c}{ SA } \\
\cline { 2 - 7 } & Ĕgitim & Test & Ĕgitim & Test & Eğitim & Test \\
\hline 2B ESA & 1.9 & 1.1 & 1.8 & 1.3 & 2.2 & 2 \\
3B ESA & 15.2 & 4.3 & 58 & 10.6 & 74 & 15.2 \\
SSRN & 56 & 12 & 86 & 18 & 89 & 18.24 \\
HybridSN & 14.1 & 4.8 & 20.3 & 6.6 & 25.5 & 9 \\
ÖY & 2.25 & 1 & 7.04 & 1.3 & 8.55 & 1.5 \\
\hline
\end{tabular}

\section{Sonuç}

Hiperspektral görüntü sınıflandırma, yüksek sınıflar arası benzerlik ve yüksek sınıf içi değişkenlik nedeniyle zorlu bir görevdir. Aynı zamanda HG sınıflandırma için uygun bir derin öğrenme modelinin tasarımı da büyük bir zorluktur. Bu çalışma kapsamında 3B ESA tabanlı MiniVGGNet yöntemi önerilmiştir. 3B ESA kullanarak, hiperspektral görüntü verilerinde bulunan uzamsal-spektral bilgilerden tam olarak yararlanmak amaçlanmıştır. MiniVGGNet kullanarak ise, eğitilebilir parametre sayısını 
azaltmak amaçlanmıştır. Parametre sayısının azalmasıyla eğitim süresi kısalmakta ve adam optimizer ağın daha iyi optimizasyonunu gerçekleştirmektedir. Önerilen yöntem, literatürde yaygın olarak kullanılan üç veri kümesinde çeşitli son teknoloji tekniklerle karşılaştırılmıştır. Önerilen yöntemin sayısal olarak daha az karmaşık olduğu ve her üç verisetinde de sınıflandırma için daha iyi bir sonuç verdiği bulunmuştur.

\section{Kaynaklar}

Ben Hamida, A., Benoit, A., Lambert, P., Ben Amar, C., 2018. 3-D deep learning approach for remote sensing image classification. IEEE Trans. Geosci. Remote Sens. 56, 4420-4434. https://doi.org/10.1109/TGRS.2018.2818945

Chen, Y.; Jiang, H.; Li, C.; Jia, X.; Ghamisi, P., 2016. Deep feature extraction and classification of hyperspectral images based on convolutional neural networks. IEEE Trans. Geosci. Remote Sens. 54, 6232-6251. https://doi.org/10.1049/iet-ipr.2019.1282

Chen, Y., Lin, Z., Zhao, X., Wang, G., Gu, Y., 2014. Deep learning-based classification of hyperspectral data. IEEE J. Sel. Top. Appl. Earth Obs. Remote Sens. 7, 2094-2107. https://doi.org/10.1109/JSTARS.2014.2329330

Firat, H., Hanbay, D., 2021. 3B ESA Tabanlı ResNet50 Kullanılarak Hiperspektral Görüntülerin Sinıflandırılması Classification of Hyperspectral Images Using 3D CNN Based ResNet50. 2021 29th Signal Process. Commun. Appl. Conf. 6-9. https://doi.org/10.1109/SIU53274.2021.9477899

Hanbay, K., 2020. Hyperspectral image classification using convolutional neural network and twodimensional complex Gabor transform. J. Fac. Eng. Archit. Gazi Univ. 35, 443-456. https://doi.org/10.17341/gazimmfd.479086

Huang, L., Chen, Y., 2020. Dual-Path Siamese CNN for Hyperspectral Image Classification With Limited Training Samples. IEEE Geosci. Remote Sens. Lett. 18, 1-5. https://doi.org/10.1109//grs.2020.2979604

Jia, J., Wang, Y., Chen, J., Guo, R., Shu, R., Wang, J., 2020. Status and application of advanced airborne hyperspectral imaging technology: A review. Infrared Phys. Technol. 104, 103115. https://doi.org/10.1016/j.infrared.2019.103115

Li, S., Song, W., Fang, L., Chen, Y., Ghamisi, P., Benediktsson, J.A., 2019. Deep Learning for Hyperspectral Image Classification: An Overview. arXiv 57, 6690-6709.

Liu, Y., Gao, L., Xiao, C., Qu, Y., Zheng, K., Marinoni, A., 2020. Hyperspectral image classification based on a shuffled group convolutional neural network with transfer learning. Remote Sens. 12, 1-18. https://doi.org/10.3390/rs12111780

Makantasis, K., Karantzalos, K., Doulamis, A., Doulamis, N., 2015. Deep supervised learning for hyperspectral data classification through convolutional neural networks. Int. Geosci. Remote Sens. Symp. 2015-Novem, 4959-4962. https://doi.org/10.1109/IGARSS.2015.7326945

Mingyi He, Bo Li, H.C., 2017. Multi-scale 3D deep convolutional neural network for hyperspectral image classification. 2017 IEEE Int. Conf. Image Process. 3904-3908.

Mohan, A., Meenakshi Sundaram, V., 2020. V3O2: hybrid deep learning model for hyperspectral image classification using vanilla-3D and octave-2D convolution. J. Real-Time Image Process. https://doi.org/10.1007/s11554-020-00966-Z

Mou, L., Ghamisi, P., Zhu, X.X., 2017. Deep recurrent neural networks for hyperspectral image 
classification. IEEE Trans. Geosci. Remote Sens. 55, 3639-3655. https://doi.org/10.1109/TGRS.2016.2636241

Paoletti, M.E., Haut, J.M., Plaza, J., Plaza, A., 2018. A new deep convolutional neural network for fast hyperspectral image classification. ISPRS J. Photogramm. Remote Sens. 145, 120-147. https://doi.org/10.1016/j.isprsjprs.2017.11.021

Roy, S.K., Krishna, G., Dubey, S.R., Chaudhuri, B.B., 2019. HybridSN: Exploring 3D-2D CNN Feature Hierarchy for Hyperspectral Image Classification. arXiv 17, 277-281.

Üzen, H., Türkoğlu, M., Hanbay, D., 2021. Derin U-Net Ağ Mimarileri Kullanarak Yüzey Hata Tespiti Surface Defect Detection Using Deep U-Net Network Architectures. 2021 29th Signal Process. Commun. Appl. Conf. https://doi.org/10.1109/SIU53274.2021.9477790

Zhong, Z., Li, J., Luo, Z., Chapman, M., 2018. Spectral-Spatial Residual Network for Hyperspectral Image Classification: A 3-D Deep Learning Framework. IEEE Trans. Geosci. Remote Sens. 56, 847-858. https://doi.org/10.1109/TGRS.2017.2755542 